\title{
The total time hypothesis, recall strategies, and memory for rapidly presented word strings
}

\author{
SHEILA M. PFAFFLIN \\ Bell Telephone Laboratories, Murray Hill, New Jersey 07974
}

\begin{abstract}
In three experiments the free recall of rapidly presented word strings was studied. The word strings were shown either in grammatical order or a scrambled order. It was found that the invariance of recall with various presentation times for grammatical sequences breaks down at presentation times between 175 and 250 msec. The order of recall, however, remains consistent with the type of sequence presented irrespective of presentation time. The amount of time required to utilize syntactic information fully in free recall is of approximately the same order of magnitude as that required to read the words.
\end{abstract}

In a number of studies it has been shown that the recall of individual words depends primarily on the total viewing time, not on how that time is distributed (Cooper \& Pantle, 1967). One of the major exceptions to the total time hypothesis is the recall of grammatical word sequences. King (1971) has shown that when grammatical sequences are presented, a word at a time, the exposure time per word does not affect recall, over a range from 5.0 to $1.0 \mathrm{sec}$. One explanation which has been offered for this difference is that the processing employed to learn grammatical sequences differs from that for random sequences, and that the former is completed in less time than the latter. If this is true, as observation time is decreased, at some point the processes by which grammatical sequences are learned must also be affected by time constraints. This point should represent the minimum time necessary to utilize syntactic information fully in the learning of sequentially presented word lists.

The order of recall is also of interest in determining what process is being employed. The free recall of unre: lated word strings shows characteristic serial position effects (Murdock, 1962). These effects are substantially modified in the case of grammatical word sequences (Deese \& Kaufman, 1957). It is of interest to ask whether the grammatical pattern of free recall shifts in the direction of that for random lists when time pressures begin to affect recall. Such a shift would suggest that $S$ s change their strategy to compensate for the loss of syntactic information. In the following experiments, the free recall of grammatical and scrambled word sequences was studied under conditions in which each word in the stimulus list was presented very briefly; the longest presentation time was $0.5 \mathrm{sec}$ per word. A fixed number of learning trials was used for the word sequences under each presentation time. The presentation times were varied to determine the effect on free recall for a range of very brief times.

\section{EXPERIMENT I}

\author{
Method
}

\section{Design}

Ss were shown four sets of eight words each. Each set was presented, one word at a time, for five trials. Two of the word sets were shown in a grammatical sequence, and two were shown scrambled. Two presentation times were used for different word sets, in combination with both scrambled and grammatical orders. A Greco-Latin square design was used to counterbalance time, word sets, and the order in which the word sets were displayed

\section{Subjects}

Ss were eight technical employees of the Bell Telephone Laboratories.

\section{Stimuli}

Four sentences of eight words each were generated. All of the words used were three-letter words selected from among the 500 most common words as given by the Thorndike-Lorge word count. There was no duplication of words in different sentences. Examples of the sentences used were "The old man has not got any job" and "Our new dog can eat one big egg."

\section{Procedure}

The Ss were run individually. They were first read instructions. The word sequences were then presented to them on an ITT Model KM 105 display oscilloscope, controlled by a DDP 116 computer. The on times for all words were set at $50 \mathrm{msec}$. Presentation times were varied by varying the time between word exposures. The off times were 200 and $450 \mathrm{msec}$. After each trial the Ss wrote down the words they recalled. Ss were allowed as much time as they wanted to recall the words. They initiated the next trial by pushing a button, and their response times were recorded. Each word sequence was shown for five consecutive trials, after which the next word sequence was begun. 


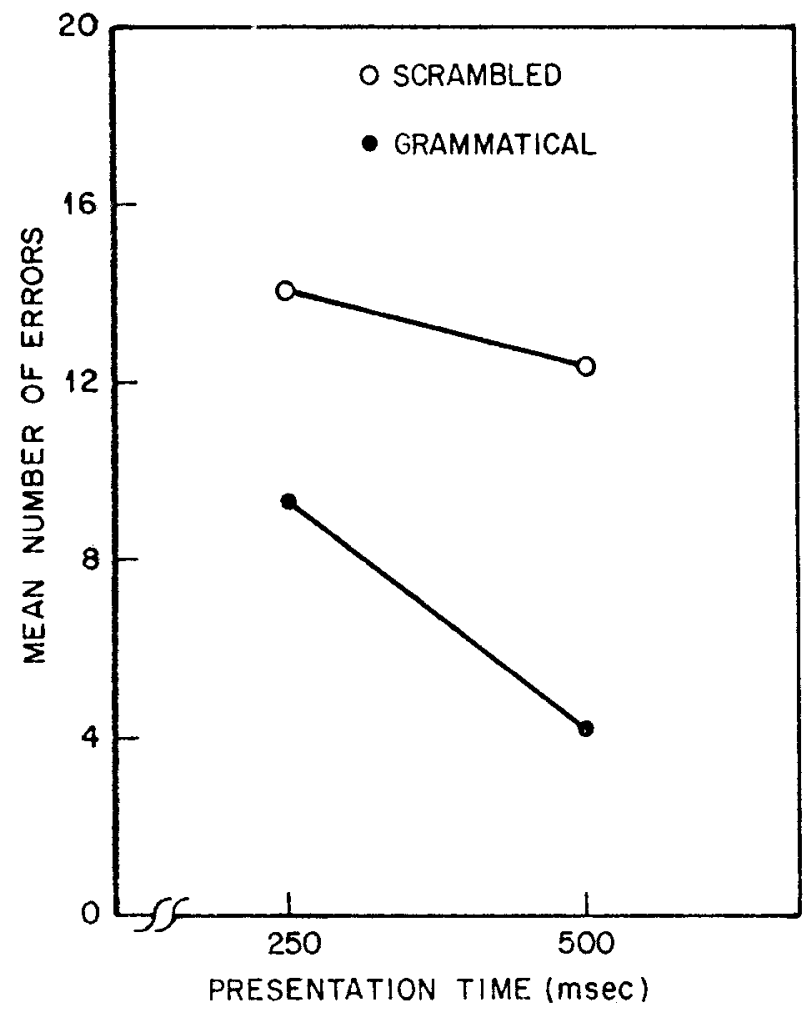

Fig. 1. Mean number of errors per $S$ for grammatical and scrambled sequences as a function of presentation time (Experiment I).

\section{Results}

The mean number of errors per $S$ for the different times is shown in Fig. 1 for grammatical and scrambled word sequences, summed over trials. As can be seen, the number of errors depends on presentation time for grammatical as well as scrambled sequences. Analysis of variance of the number of errors, summed over trials, shows that the difference between grammatical and scrambled sequences is significant, $F(1,4)=83.25$, $\mathrm{p}<.001$. The time variable is also significant, $F(1,4)=$ $18.45, p<.025$. The interaction of the two variables is not significant.

The response times were also examined by analysis of variance. Response time was found to vary with the order in which the word sets were shown, $F(3,75)=$ $4.25, \mathrm{p}<.005$, and with trial, $\mathrm{F}(4,75)=7.64, \mathrm{p}<.001$, but not with time or type of sequence. Response times increase with the number of trials; it increases, then decreases, with word set order.

\section{Discussion}

The results of this study show that recall is a function of observation time for grammatical word sequences when the time is sufficiently short. However, the difference in increase in number of errors for the two types of sequences, though in the expected direction of a greater change for grammatical sequences, was not significant. The response times showed the expected relationship to amount recalled, except in the case of the type of sequence. Apparently, Ss spend more time recalling each word for the scrambled sequences.

The limited number of times used in this experiment were insufficient to study the relationship of time and recall of various sequences in any detail. Therefore, another experiment was run to study this question further.

\section{EXPERIMENT II}

\section{Method}

Design

Ss were shown eight sets of eight words each. Each set was presented, one word at a time, for five trials. Four sets were presented in grammatical order, and four in scrambled order, to each S. Four different times were combined with the sequences. A Greco-Latin square design was used to balance time and type of sequence, word set, and word set order effects.

\section{Subjects}

Ss were 16 technical employees of the Bell Telephone Laboratories.

\section{Stimuli}

Eight sentences of eight words each were generated. The words ranged in length from two to six letters, and were selected from among the 500 most common words as given by the Thorndike-Lorge word count. There was no duplication of words in different sentences.

\section{Procedure}

The procedure was similar to that used in the previous study, except that the response time was fixed at $30 \mathrm{sec}$. The on times for the words were held constant at $50 \mathrm{msec}$. The off times were $50,125,200$, and $275 \mathrm{msec}$.

\section{Results}

The mean number of errors per $\mathrm{S}$ for all trials for grammatical and scrambled sequences is given in Fig. 2 as a function of total exposure time. The application of analysis of variance to these data is complicated by the very low error rates for the grammatical sequences at longer times. Therefore, the error data for the different times were analyzed for each type of sequence by means of the Friedman two-way analysis of variance by ranks. When all four times were included, the tests were significant for both types of sequences $(p<.001)$. When the analysis was redone, omitting the shortest time, the result was not significant for the scrambled sequences. It was also not significant for the grammatical sequences when all the data were used. However, several Ss made no errors for these times. When these Ss were dropped from the analysis, the test was significant for the remaining data $(\mathrm{p}<.05)$.

The average recall position values of the words from 
various presentation positions are plotted in Fig. 3 for the grammatical and scrambled sequences. There is a linear relationship between original position and mean recall position for both scrambled and grammatical sequences.

\section{Discussion}

The results again show that recall is a function of presentation time for grammatical sequence when the time per word becomes short enough. However, the pattern of the decline was somewhat unexpected. In the case of the scrambled sequences, additional time was not facilitating except for the earliest increase. However, additional time results in additional improvement for the grammatical sequences. Thus, it appears that the grammatical sequences come closer than the scrambled sequences to conforming to the total time hypothesis in this study.

Examination of the order of recall reveals a tendency to recall words in the order in which they are presented for both grammatical and scrambled sequences. This order is characteristic of grammatical sequences in other free recall studies, but is unusual for random word sequences, which usually show a tendency for the words presented last to be recalled first. If Ss are adopting a strategy which is suitable only for grammatical

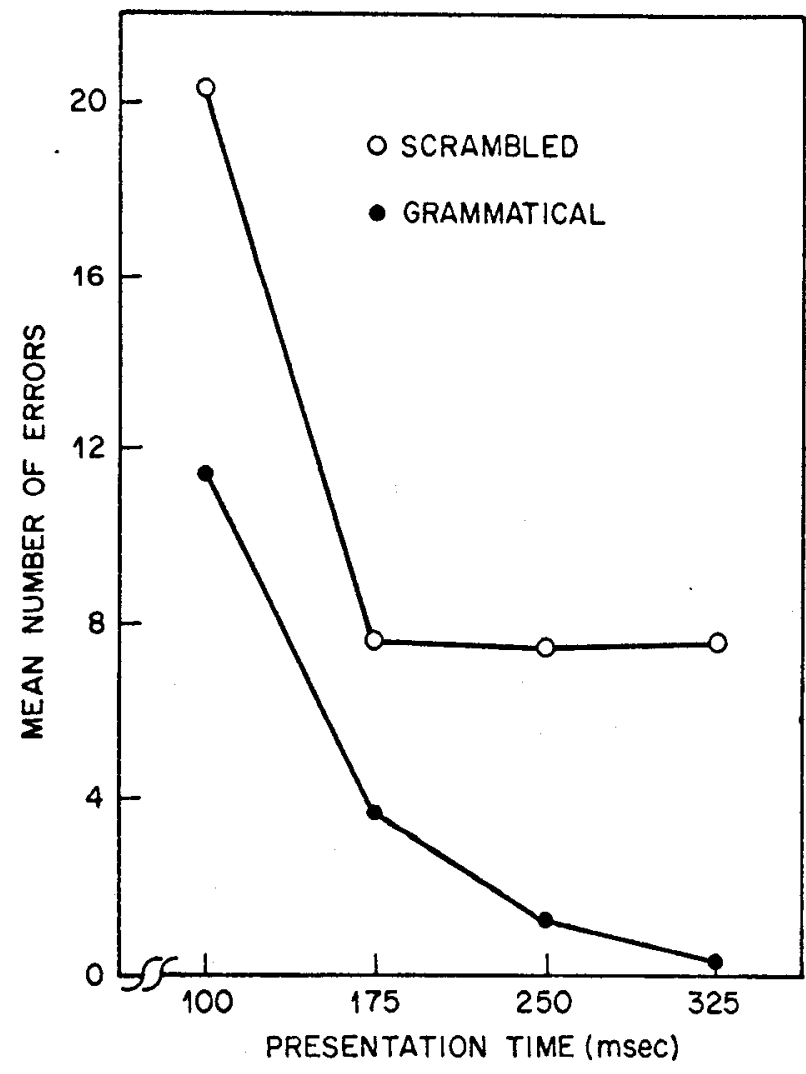

Fig. 2. Mean number of errors per $S$ for grammatical and scrambled sequences as a function of presentation time (Experiment II).

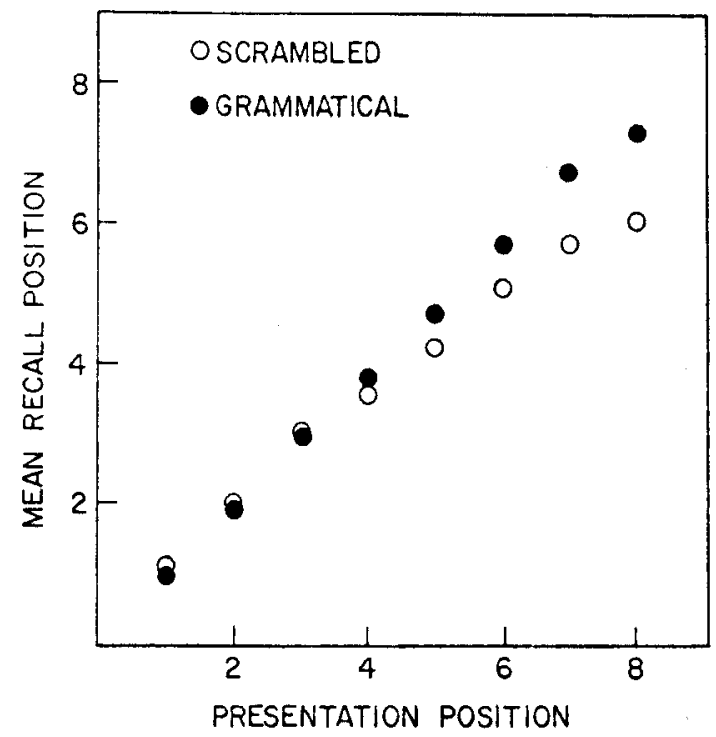

Fig. 3. Mean recall position of responses from various presentation positions for grammatical and scrambled sequences (Experiment II).

sequences and applying it to both types of sentences, it may explain the anomalous findings in regard to the total time hypothesis for the scrambled sequences.

The short word sequences used in this experiment, which barely exceed the memory span, may also have contributed to the findings discussed above. It is possible that some Ss may have behaved as though the experiment were a series of memory span tests, rather than attempting to learn the material. Another experiment was therefore run in which the length of the sequences was increased, and Ss received only one type of sequence.

\section{EXPERIMENT III}

Method

Design

Two groups of Ss were run, one for the grammatical sequences and one for the scrambled sequences. Four presentation times were used combined with four word sets and run as within-S variables. A Greco-Latin square design was used to counterbalance the effects of time, word set, and word set order.

\section{Subjects}

Ss were 16 students from a local high school. They were paid volunteers.

Stimuli

Four lists of 16 words were generated by pairing the eight word sets of Experiment II, with the first 8 words in the combined list coming from one set and the last 8 from the other set. The order of the words within sets was the same as Experiment II.

\section{Procedure}

The procedure was similar to that used in Experiment II, with 


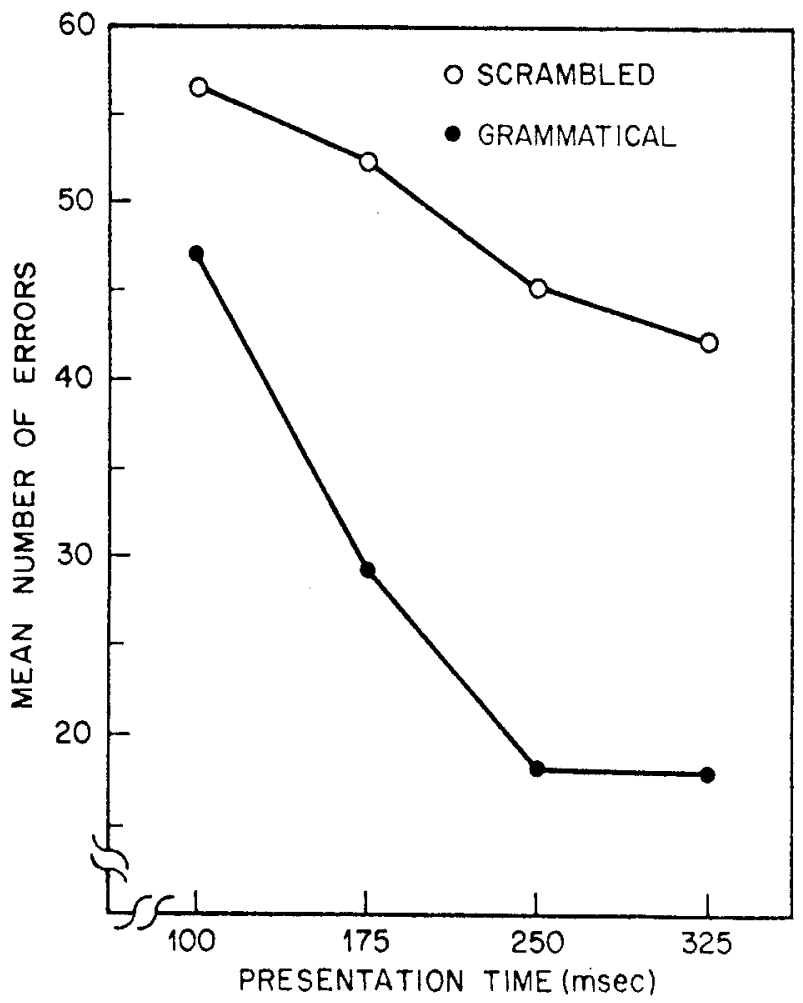

Fig. 4. Mean number of errors per $S$ for grammatical and scrambled sequences as a function of presentation time (Experiment III).

a fixed time for responding of $60 \mathrm{sec}$. Prior to the start of the next trial, the trial number appeared on the screen to warn the $S$ that a trial was to start. The times were the same as in Experiment II.

\section{Results}

The mean number of errors per $S$ summed over trials is shown in Fig. 4. Analysis of variance of the error data indicated a significant interaction between time and type of sequence, $F(3,150)=27.77$. As can be seen, the predicted relationship between time and number of errors is found for the two types of sequences. Number of errors is a linear function of observation time for the random sequences. There is virtually no difference for the two longer times for the grammatical sequences, and then a rapid increase in number of errors for two shorter times.

The order in which the words are recalled is plotted in Fig. 5. The rank order correlation between presentation position and mean recall position is 0.96 for the grammatical sequences, which is significant beyond the 0.01 level. This correlation is not significant for the scrambled sequences.

In order to determine whether Ss shift their order of recall for grammatical sequences in the direction of that for scrambled sequences under time constraint, Spearman rank order correlations between average recall position and presentation position were computed on grammatical and scrambled sequences for the 100-msec time. The correlation for the grammatical sequences is 0.96 , significant beyond the 0.01 level. For the scrambled sequences it is 0.49 , which is not significant.

\section{GENERAL DISCUSSION}

The results of these experiments show that exposure time affects recall of grammatical sequences when the time becomes sufficiently short. However, the time at which this occurs is short enough so that visual processes associated with the previous word may still be active when the next word is shown (Averbach \& Coriell, 1960). It may, therefore, be asked whether the increase in errors at short times is due to interference with grammatical processing or whether the Ss are unable to see the words due to the masking effect of the next stimulus. The fact that interference with visual processing may occur does not in itself supply the answer to this question. The presentation of the next stimulus presumably interferes with processing of the previous stimulus for any time interval. The relevant question is whether the words could be reported as seen if there were no memory load on the Ss. Two lines of evidence may be offered to support the view that masking does not prevent the Ss from seeing the words even at the shortest time interval. First, studies have been done which indicate that Ss can see five-letter words in $100 \mathrm{msec}$ under conditions similar to our experiment (Haber, 1970). Since most of our words were five letters or less, masking should have had a minor effect at $100 \mathrm{msec}$ and none at $175 \mathrm{msec}$. Furthermore, if the rate of presentation was interfering

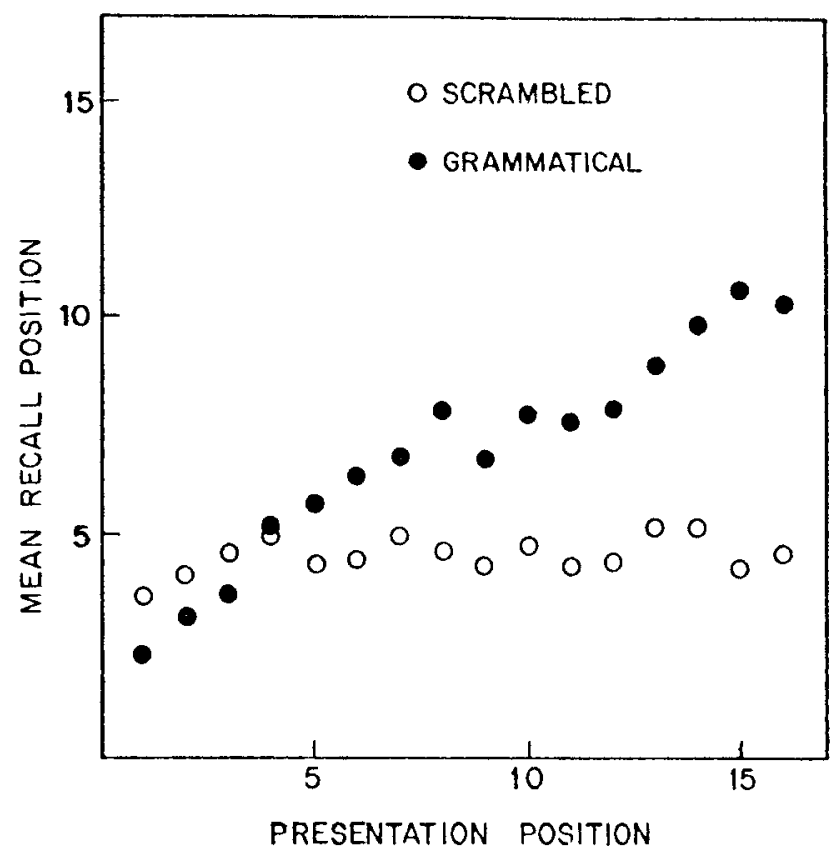

Fig. 5. Mean recall position of responses from various presentation positions for grammatical and scrambled sequences (Experiment III). 
with the S's ability to see the stimuli, both grammatical and scrambled sequences should have been affected. However, the decrease in errors for increasing observation times is approximately linear for the scrambled sequences over the four time intervals in Experiment III. It therefore appears that the increase in number of errors between 175 and $250 \mathrm{msec}$ for the grammatical sequences represents disruption of syntactic processing rather than the effect of interference with visual processing.

It is of interest to note that these times fall in the same region as average fixation times found for reading. It is true that in reading, more words are processed per fixation than are being processed per presentation in our experiments. However, it is likely that some parallel processing occurs in reading. Thus, it appears that Ss under instructions to learn grammatical sequences effectively utilize an amount of time which is not greatly in excess of that normally used to read the words. Learning of grammatical sequences may, in fact, be equivalent to reading them, at least insofar as the time required is concerned.

This result does not, of course, rule out the possibility that learning occurs in parallel with reading, though in this case it is not clear how the learning process takes advantage of grammatical constraints. Also, the single word presentation used does allow some extra time for learning. Additional research using multiple word presentations might clarify the matter. However, if additional processing of grammatical sequences is done under learning instructions, it takes place in far less time than learning of scrambled sequences.

\section{REFERENCES}

Averbach, E., \& Coriell, A. S. Short-term memory in vision. Bell System Technical Journal, 1961, 40, 309-328.

Cooper, E. H., \& Pantle, A. J. The total-time hypothesis in verbal learning. Psychological Bulletin, 1967, 68, 221-234.

Deese, J., \& Kaufman, R. A. Serial effects in recall of unorganized and sequentially organized verbal material. Journal of Experimental Psychology, 1957, 54, 180-187.

Haber, R. N. How we remember what we see. Scientific American, 1970, 222, 104-112.

King, D. J. Influence of interitem interval in the learning of connected discourse. Journal of Experimental Psychology, 1971, 87, 132-134.

Murdock, B. B.; Jr. The serial position effect of free recall. Journal of Experimental Psychology, 1962, 64, 482-488.

(Received for publication August 3, 1973; accepted August 27, 1973.) 